\title{
Power Load Event Detection and Classification Based on Edge Symbol Analysis and Support Vector Machine
}

\author{
Lei Jiang, ${ }^{1}$ Jiaming Li, ${ }^{2}$ Suhuai Luo, ${ }^{1}$ Sam West, ${ }^{3}$ and Glenn Platt ${ }^{3}$ \\ ${ }^{1}$ School of DCIT, University of Newcastle, Callaghan, NSW 2308, Australia \\ ${ }^{2}$ ICT Centre, Commonwealth Scientific and Industrial Research Organization, Clayton South, VIC 3169, Australia \\ ${ }^{3}$ Energy Technology Division, Commonwealth Scientific and Industrial Research Organization, Clayton South, VIC 3169, Australia
}

Correspondence should be addressed to Lei Jiang, lei.jiang@csiro.au

Received 30 April 2012; Revised 8 August 2012; Accepted 18 October 2012

Academic Editor: F. Morabito

Copyright (C) 2012 Lei Jiang et al. This is an open access article distributed under the Creative Commons Attribution License, which permits unrestricted use, distribution, and reproduction in any medium, provided the original work is properly cited.

\begin{abstract}
Energy signature analysis of power appliance is the core of nonintrusive load monitoring (NILM) where the detailed data of the appliances used in houses are obtained by analyzing changes in the voltage and current. This paper focuses on developing an automatic power load event detection and appliance classification based on machine learning. In power load event detection, the paper presents a new transient detection algorithm. By turn-on and turn-off transient waveforms analysis, it can accurately detect the edge point when a device is switched on or switched off. The proposed load classification technique can identify different power appliances with improved recognition accuracy and computational speed. The load classification method is composed of two processes including frequency feature analysis and support vector machine. The experimental results indicated that the incorporation of the new edge detection and turn-on and turn-off transient signature analysis into NILM revealed more information than traditional NILM methods. The load classification method has achieved more than ninety percent recognition rate.
\end{abstract}

\section{Introduction}

Nowadays, multifarious classification techniques are widely used in many signal-processing areas, such as face recognition, road traffic analyze, medical image, weather forecasting, and so forth $[1,2]$. It has been considered whether this kind of techniques could serve the purposes of power load monitoring, which is a process for obtaining what appliances are used in the house as well as their individual energy consumption by analyzing changes in the voltage and current. In particular, information about the operating conditions of consumers (such as what appliances are operated and what state they are in) is useful for demand estimation and prediction. In addition, the operating conditions of electrical appliances in modern society clearly reflect consumers' lifestyles and behavior patterns [3]. Research is being performed on the use of such information for circuit diagnoses, safety confirmation systems for elderly persons living alone [4], demand management, and optimization [5-10], and for other uses [11].
There are mainly two classes of approaches in power monitoring algorithm, including intrusive appliance load monitoring (IALM) and nonintrusive appliance load monitoring (NIALM) [12]. In order to measure consumption, IALM distributes direct sensors at each device or appliance. Although conceptually straightforward and potentially highly accurate, direct sensing is often expensive due to time consuming installation and the requirement for one sensor for each device or appliance. In response to limitations with the direct sensing approach, researchers have explored methods to infer energy usage via a single sensor.

In contrast to the direct sensing methods, the standard NIALM configuration includes a sole sensor set to measure current and voltage, as well as a processing algorithm for determining the status of various devices [13]. It was firstly studied by Hart of MIT in the early 1990s with funding from the Electric Power Research Institute [12]. Since then, different strategies for NIALM have been developed. One of the important approaches on classification is to apply support vector machine (SVM) [14-18] in the nonintrusive 
appliance load monitoring. It is imperative for power system research field to evaluate the SVM on this task from a practical point of view. As the preprocess for finding out the features for SVM, transient detection also plays an important role in NIALM.

In order to decompose the total loads into their components, Bijker et al. employed different power level, or named step change for the purpose [19]. Chang et al. used DWT and a new method coreless HCT to detect the power events [20]. Nonetheless, they only supposed to detect the events of appliances with complicated features, such as motor, microwave oven, and thyristor rectifier. In 2002, Onoda et al. used the data proposed in [21] for the purpose of estimating the state of household electric appliances. They compared different types of SVMs obtained by choosing different kernels. They reported results of polynomial kernels, radial basis function kernels, and sigmoid kernels. All results for the three different kernels achieved almost same error rates. However, in the estimation of the state of household electric appliances, the results for the three different kernels achieved different error rates. They also compared different capacity of SVMs obtained by choosing different regularization constants and parameters of kernels experimentally. The results showed that the capacity control is as important as the choice of kernel functions. Kadouche and his colleagues later presented [22] their ongoing work on the house occupant prediction issue based on daily life habits in smart houses. Most of their works were based on supervised learning technical. They used SVM to build behavior classification model for learning the user's habits, analyzed the publicly available dataset from the Washington State University Smart Apartment Test-bed. Particularly, they evaluated the grooming, having breakfast, and bed to toilet activities [23]. Their experimental results showed that the user can be recognized with a high precision which means that each user has his own way to perform activities. As future work, the users' patterns which allow a person to be discriminated and recognized among a group, performing multiactivities in the same environment without using intrusive technologies were being studied.

Grinblat and his fellows presented a new method for generating adaptive classifiers and capable of learning concepts that change with time, which is the time-adaptive support vector machine (TA-SVM) [24]. The basic idea of TA-SVM is to use a sequence of classifiers, each one is appropriate for a small time window but, in contrast to other proposals, learning all the hyperplanes in a global way. Starting from the solution of independent SVMs, they showed that the addition of a new term in the cost function (which penalizes the diversity between consecutive classifiers) produces in fact a coupling of the sequence. Once coupled, the set of SVMs acts as a single adaptive classifier. They evaluated different aspects of the TA-SVM using artificial drifting problems. In particular, they showed that changing the number of classifiers and the coupling constant can effectively regularize the sequence of classifiers. They compared TA-SVM with other state-of-the-art methods in three different settings: estimation, prediction, and extrapolation, including problems with small datasets, high-dimensional input spaces, and noise. TA-SVM showed in all cases to be equivalent to or better than the other methods. Even for the most unfavorable situation for TASVM, that is, the sudden changes of the dataset, their new method showed a very good performance. However, the limitation of this method may be reflected in the long drifting time and the requirement of additional hardware. A more recent research of Liang's group [25], combined various features including current waveform (CW), active/reactive power (PQ), harmonics (HAR), instantaneous admittance waveform (IAW), instantaneous power waveform (IPW), eigenvalues (EIG), and switching transient waveform (STW). The results of their research provided a higher degree of recognition precision, but the algorithm requires mountains of work on collecting and processing appliance signatures.

In this paper, the data collected in real world is used and clearly analyzed what are the important issues for applying SVM to power system research field. The remainder is organized as below. Section 2 gives the details of the new transient event detection process named ESA and the reasons why we choose SVM as our method and the main process of load classification. The details of data collecting and preprocessing along with the experiment results are shown in Section 3. Finally, conclusions are made in Section 4.

\section{Power Load Events Detection and Classification}

It is known that although NIALM based on different techniques, it has several common principles [26].

(i) Load features classification: specific appliance features, or signatures, need to be selected and mathematically characterized.

(ii) Mechanism: a hardware installation (sensor and data acquisition system) that can detect the selected features is required.

(iii) Decomposition: a mathematical algorithm detects the features in the overall signal and output.

Based on this theory, Figure 1 describes the process of this research. The mechanism collects the whole operation of a circuit, then, the appropriate algorithms draw out all the electrical events in the circumstance. After successfully seeking the point "load $x$ switch on," it is feasible to classify load $x$, afterwards seek the point "load $x+1$ switch on" excluding faked edges generated by previous loads. The same procedure subsequently goes for searching switch off events.

\subsection{Power Load Events Detection Using Edge Symbol Analysis Method}

2.1.1. Background. Methods of edge detection have been widely studied in many areas, primarily developed in the discrete cases and have been confined to slightly noisy signals. The regularization or smoothing and optimal approaches of Canny [27] have led to several efficient continuous operators for noisy and blurred signals. Other advanced methods that 


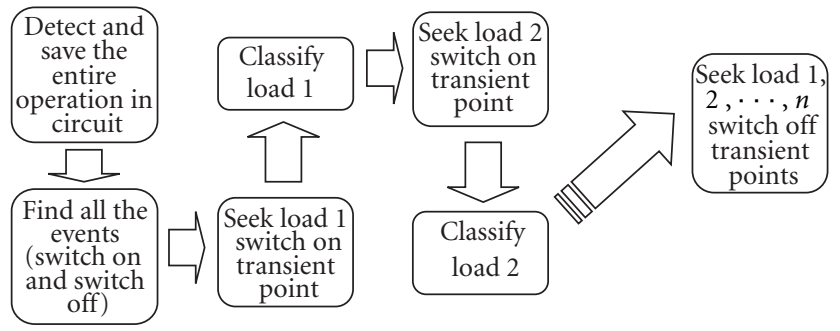

FIGURE 1: The load events detection procedure.

consider the Canny criterion have been developed to deal with noise, uneven illumination, and image contrast. In a marginal way, discrete approaches for regularization have been developed and have improved results by considering the discrete nature of the signals $[28,29]$.

Introducing nonlinearity into the global filtering process, as in noisy edge detection, is a marginal yet efficient method of obtaining good performance. Generally, nonlinear filtering is used in a preliminary regularization stage. Pitas and Venetsanopoulos proposed a class of nonlinear filters that reject additive and impulse noise, while preserving the edges [30]. More recently, Benazza-Benyahia et al. introduced a nonlinear filter bank leading to a multiresolution approximation of the signal in which discontinuities are well preserved [31]. A nonlinear filter for edge enhancement, using a morphological filter, has been proposed by Schulze [32]. The author showed that local variation analysis allows enhancing edges corrupted by multiplicative noise. Hwang and Haddad presented an integrated nonlinear edge-detectionbased denoising scheme [33]. A thresholded derivative is computed from two half filters (median for impulse noise, mean for Gaussian noise, and min-max for uniform noise) and edge detection is used to select the second filtering stage, that is, mean for noise or median for edge points. In the scheme, edge detection could be considered as a by-product and the optimal performance is obtained only when the correct first filter is selected according to the noise statistic. Based on these theories, Laligant and his colleague propose to obtain both noise reduction and edge detection by a one-stage nonlinear derivative scheme [34]. The scheme, which consists of combining two polarized differences, yields significant improvements in signal-tonoise ratio without using regularization or increasing the computational requirements.

2.1.2. The Approach. It is known that three kinds of slopes can be found in all power waveforms, including positive, negative, and constant. So the edge symbol analysis method (ESA) can find the position of the edge point according to the sign of the signal area. In this way, the edge point will be located after the transition if the slope is positive. Otherwise, when it is negative, the edge point will be located before the slope. Here, for the sake of detecting these two kinds of edges, two detector filters $L_{+}$and $L_{-}$have to be introduced. Since $L$ is chosen as an antisymmetric linear filter, which gives the localization changes and this shifted pixel localization depending on edge's orientation. These filters have the relationship described in (1).

$$
L_{-}(S)=-L_{+}\left(S^{-1}\right) \text {. }
$$

With the simple threshold $H$, we use the combination of $L_{+}$ and $L_{-}$as below to detect edge points of the original input signal $I$.

$$
\begin{gathered}
O_{+}(S)=H\left[L_{+}(S) I(S)\right], \\
O_{-}(S)=-H\left[-L_{-}(S) I(S)\right] .
\end{gathered}
$$

2.2. SVM-Based Power Load Classification. The proposed power classification method is composed of two major stages, including: first a frequency feature analysis is applied on current signal, and then a trained classifier based on SVM is applied to identify different appliances. The following sections give the details of the approach.

2.2.1. Current Feature Analysis. To save computational resources and improve performance, only current signals were used for frequency analysis with fewer sample points [35]. For example, according to previous experiments, the accuracy rate of the classification is almost the same or even higher when employing 500 frequency signal points as features instead of 2000 unprocessed current signal points.

After collecting the data of single device and extracting the current signals, a short-time fast Fourier transform (FFT) of the signal is performed as in (3). Where $\omega_{N}=e^{(2 \pi i) / N}$ is an $n$th root of the unity.

$$
\begin{gathered}
X(k)=\sum_{j=1}^{N} x(j) \omega_{N}^{(j-1)(k-1)}, \\
x(j)=\left(\frac{1}{N}\right) \sum_{k=1}^{N} X(k) \omega_{N}^{-(j-1)(k-1)},
\end{gathered}
$$

and an example is shown as Figure 2.

2.2.2. SVM-Based Classification. SVM, as one of the method of NIALM system, is relatively insensitive to the number of data points and the classification complexity does not depend on the dimensionality of the feature space [36]. Therefore, it can potentially learn a larger set of patterns and be able to scale better than some other methods. Once the data are classified into two classes, a suitable optimizing algorithm can be used if necessary for further feature identification, depending on the application.

Support vector machine is a training algorithm for learning classification and regression rules from data SVM was first introduced by Cortes and Vapnik [37] in the 1990s for classification and have recently become an area of intense research owing to developments in the techniques and theory coupled with extensions to regression and density estimation. It is based on the structural risk minimization principle which incorporates capacity control to prevent overfitting. It is a partial solution to the bias-variance tradeoff dilemma [38]. It has been widely used in different areas. 

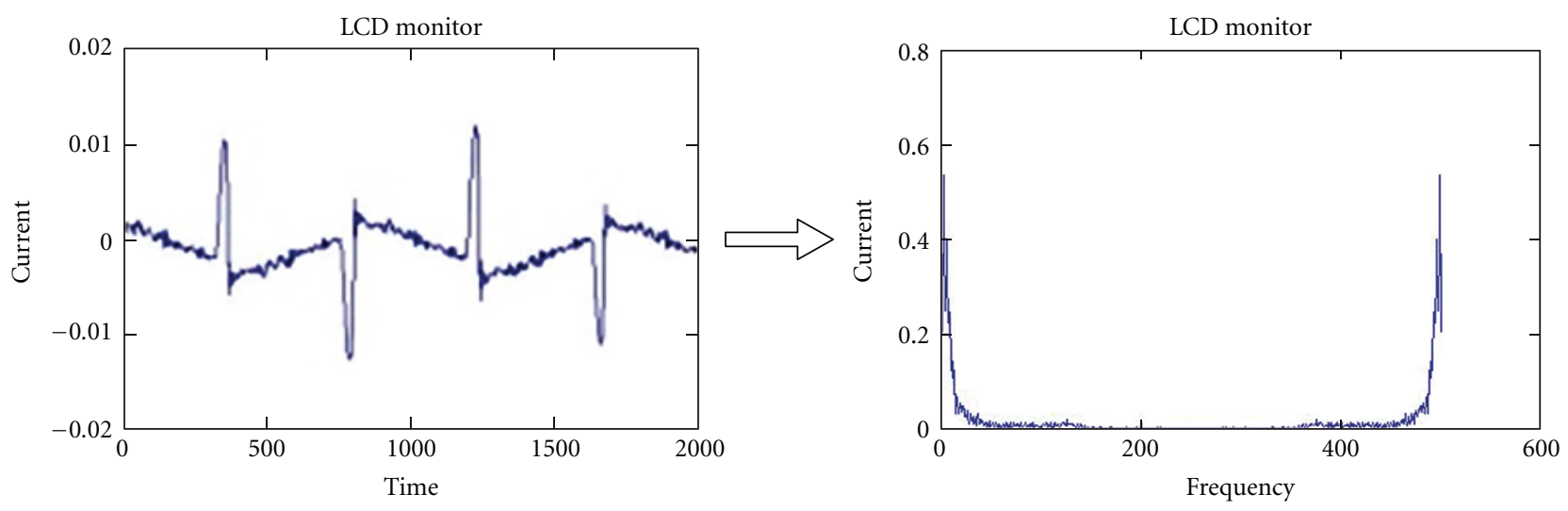

FIGURE 2: Load current signal of LCD monitor in time and frequency domains.

As a classifier, it gives a set of training examples, each marked as belonging to one of two categories. The SVM training algorithm builds a model that predicts whether a new example falls into one category or the other. Intuitively, an SVM model is a representation of the examples as points in space, mapped so that the examples of the separate categories are divided by a clear gap that is as wide as possible [39]. New examples are then mapped into that same space and predicted to belong to a category based on which side of the gap they fall on.

SVM delivers state-of-the-art performance in real-world applications such as text categorization, hand-written character recognition, biosequences analysis, image classification, and so forth. It is now established as one of the standard tools for machine learning and data mining. The SVM decision function is defined as (4)

$$
f(y)=\sum_{i=1}^{N} \alpha_{i} K\left(x_{i}, y\right)+b .
$$

Here, $y$ is the unclassified tested vector, $x_{i}$ is the support vectors and $\alpha_{i}$ is their weights, and $b$ is a constant bias. $K(x, y)$ is the kernel function introduced into SVM to solve the nonlinear problems by performing implicit mapping into a high-dimensional feature space [40].

Consider the problem of separating the set of training vectors belonging to two separate classes [41].

$$
D=\left\{\left(x^{1}, y^{1}\right), \ldots,\left(x^{l}, y^{l}\right)\right\}, \quad x \in R^{n}, y \in\{-1,1\}
$$

with a hyperplane,

$$
\langle\omega, x\rangle+b=0
$$

The kernel function chosen in our algorithm is Gaussian

$$
k\left(x_{i}, x_{j}\right)=\exp \left(-\gamma\left\|x_{i}-x_{j}\right\| 2\right), \quad \text { for } \gamma>0,
$$

where scalar $\gamma$ is identical for all coordinates.
TABle 1: Data collecting details.

\begin{tabular}{ll}
\hline Load & Description \\
\hline Dremel & $\begin{array}{l}\text { High speed electric motor (a Dremel is a } \\
\text { handheld tool for grinding, engraving, etc.). } \\
\text { Has built-in speed control which modifies } \\
\text { the current sine wave in all except full speed } \\
\text { settings. }\end{array}$ \\
& $\begin{array}{l}\text { Small fan heater with fan, medium heat, and } \\
\text { high heat modes selected using a slide switch } \\
\text { Fan heater }\end{array}$ \\
2-fanspeed digital temperature controlled \\
heat gun & $\begin{array}{l}\text { A normal kettle } \\
\text { Kettle }\end{array}$ \\
Laptop charger & $\begin{array}{l}\text { Dell laptop charger for a D630 laptop } \\
\text { ACD monitor }\end{array}$ \\
Incandescent light & $\begin{array}{l}\text { Incandescent light bulb in small desk lamp } \\
\text { Circular fluorescent tube light from }\end{array}$ \\
Fluorescent light & soldering station magnifying light stand \\
Mini PC & $\begin{array}{l}\text { Dream PC (labeled PGWY1) } \\
\text { A normal personal computer }\end{array}$ \\
PC & A cheap \$15 3-speed plastic pedestal fan \\
Pedestal fan &
\end{tabular}

\section{Experiments}

3.1. Data Collecting and Preprocessing. All the data are collected in Commonwealth Scientific and Industrial Research Organization (CSIRO) and a residential house in Newcastle, NSW, Australia. Collecting details are shown in Table 1.

Figure 3 shows the segmentation process in this experiment. Each signal of load has three phrases: on phrase, steady state, and off phrase.

\subsection{Experiment Result and Discussion}

3.2.1. Implementation of ESA. In Figure 4, the overlapped data of Kettle and Toaster are shown as an example. Figure $4(\mathrm{a})$ is the original overlapped data of kettle and oven. The red figures in Figure 4(b) are the output of edge detection and the original data. The on and off edges are represented in red color. To clearly observe the resultant edge 


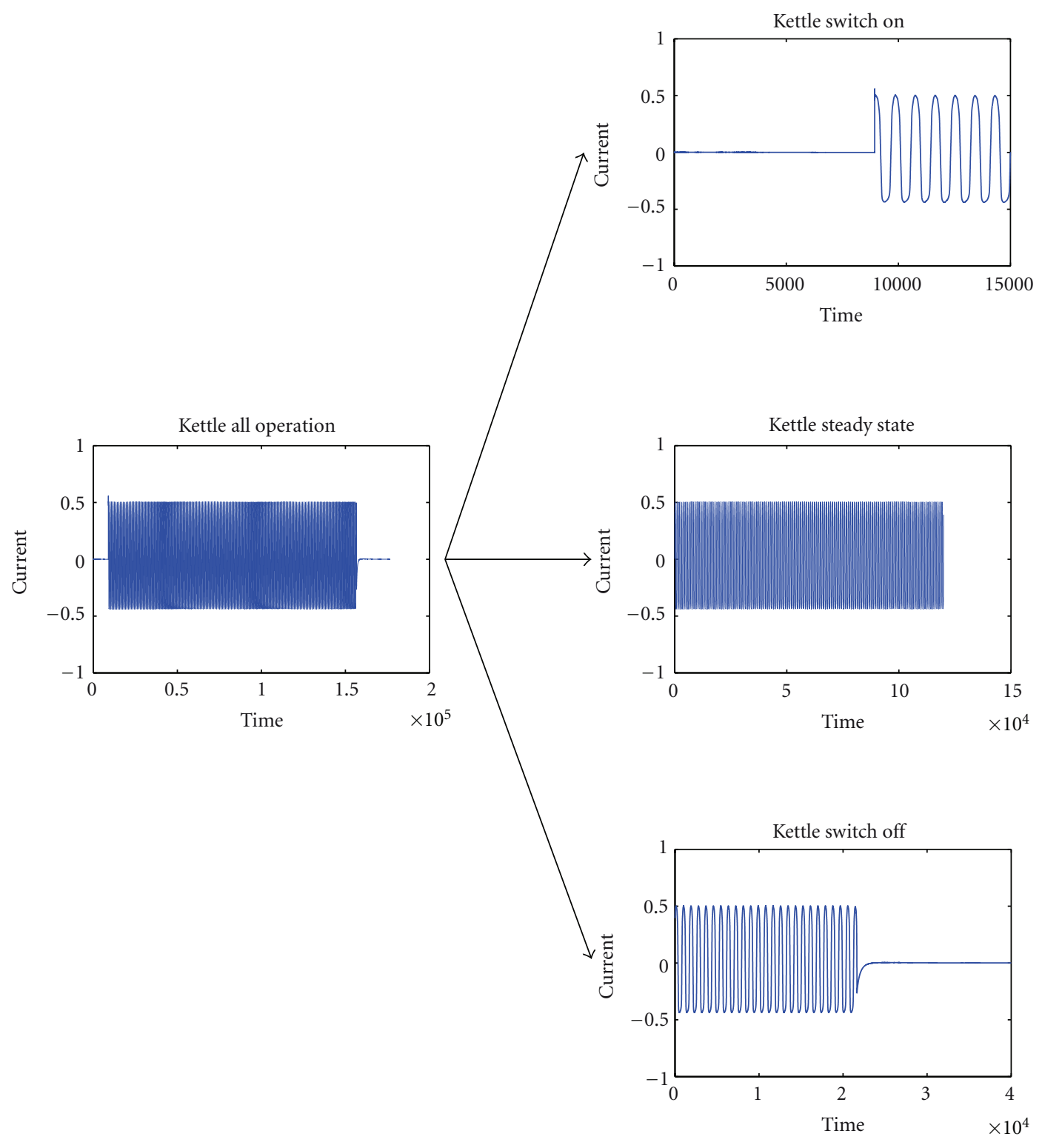

FIgURe 3: Process of signal segmentation.

points, the edge points in Figure 4(b) (i.e., the red curve) are redrawn in Figure 4(c).

The example of overlapping kettle with microwave oven (Figure 5(a)) and PC (Figure 5(b)) is shown in Figure 5. It can easily find the edges (as the yellow arrows shown in Figure 5) by repeating the process that mentioned above, and then relocate the transient points in the original data. When the transient features are recognized as "microwave oven on" or "PC on" by SVM, the algorithm ignores the next "Off" and "On" events because they are fake symbols. It is thus able to obtain the full process of the whole event.

Data of 2 loads combinations from four typical appliances are employed for testing in this experiment. The algorithm runs on 10 different data for each group. As for a single case, the performance time consumption is lower than 4 seconds. The result of this process is shown in Table 2.
Practically, all events are accurately detected by ESA except the Load2-On, whereas the accuracy is still higher than $80 \%$.

3.2.2. Implementation of Load Classification. The recognition process on each phrase is running with Gaussian as the function kernel. Using the segmented steady-state power data for the test, some selected features are shown in Figures 6 and 7, where Figure 6 shows the training unprocessed current data in time domain, and Figure 7 shows the training current data in frequency domain. The algorithm runs 70 times on the data collected from the 11 different loads. As for a single case, the performance time consumption is lower than 2 seconds. The recognition results are shown in Table 3.

It can be seen that all the recognition accuracy rates are higher than $90 \%$. It shows the ideal recognition performance 


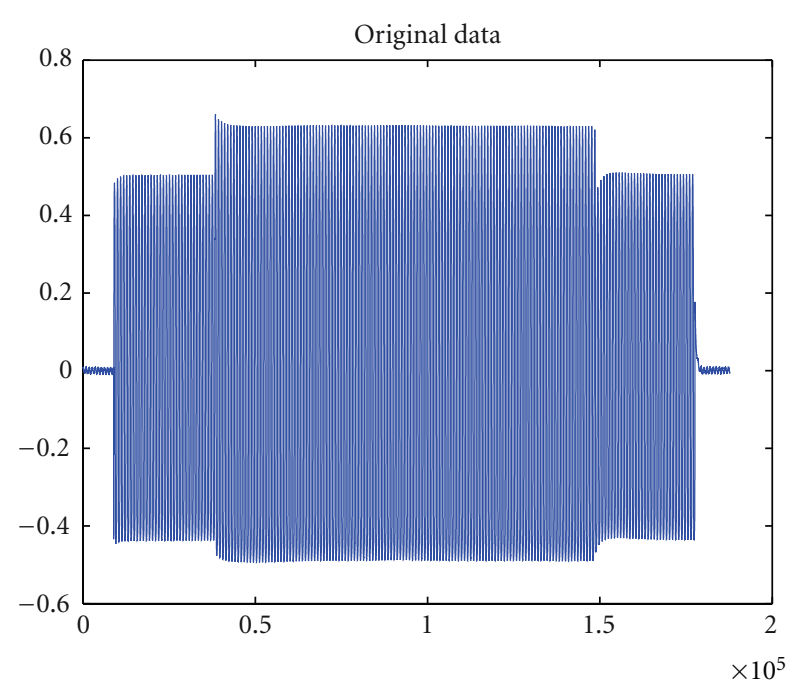

(a)

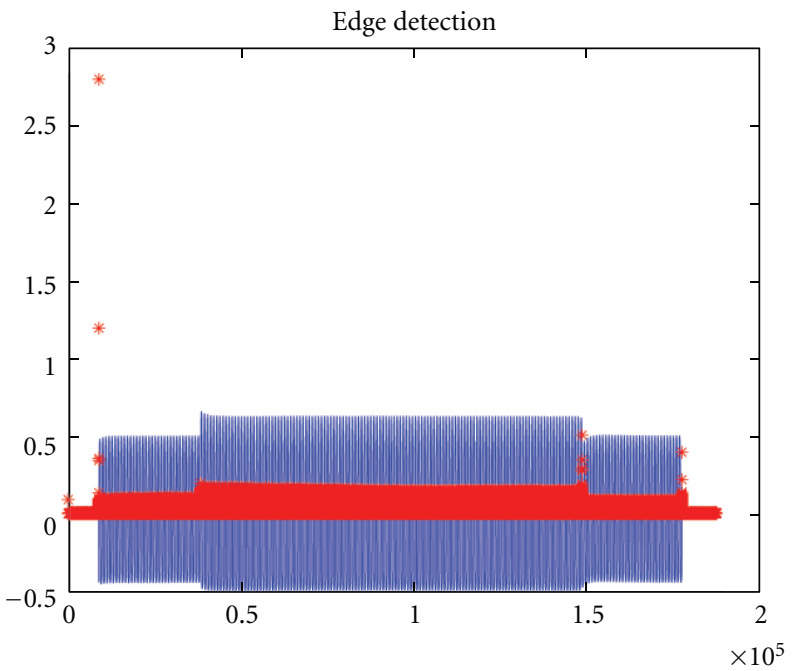

(b)

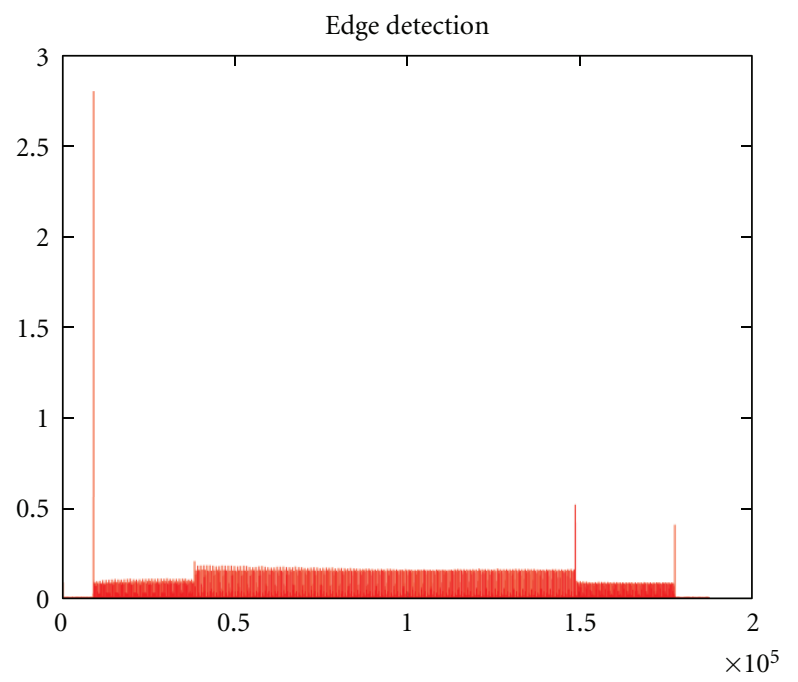

(c)

Figure 4: The original data and edge analysis of Kettle + Oven.

TABLE 2: Result of ESA on four typical loads.

\begin{tabular}{|c|c|c|c|c|}
\hline \multirow{2}{*}{ Overlapped load appliances groups } & \multicolumn{4}{|c|}{ Accuracy of recognizing transient events (switch on and switch off) $\%$} \\
\hline & Load 1 On & Load 2 On & Load 2 Off & Load 1 Off \\
\hline Kettle + Oven & 100 & 100 & 100 & 100 \\
\hline Oven + Kettle & 100 & 100 & 100 & 100 \\
\hline Kettle + Microwave oven & 100 & 80 & 100 & 100 \\
\hline Microwave oven + Kettle & 100 & 90 & 100 & 100 \\
\hline Kettle + PC & 100 & 100 & 100 & 100 \\
\hline PC + Kettle & 100 & 90 & 100 & 100 \\
\hline Oven + Microwave oven & 100 & 100 & 100 & 100 \\
\hline Microwave oven + Oven & 100 & 80 & 100 & 100 \\
\hline Oven + PC & 100 & 100 & 100 & 100 \\
\hline $\mathrm{PC}+$ Oven & 100 & 80 & 100 & 100 \\
\hline
\end{tabular}



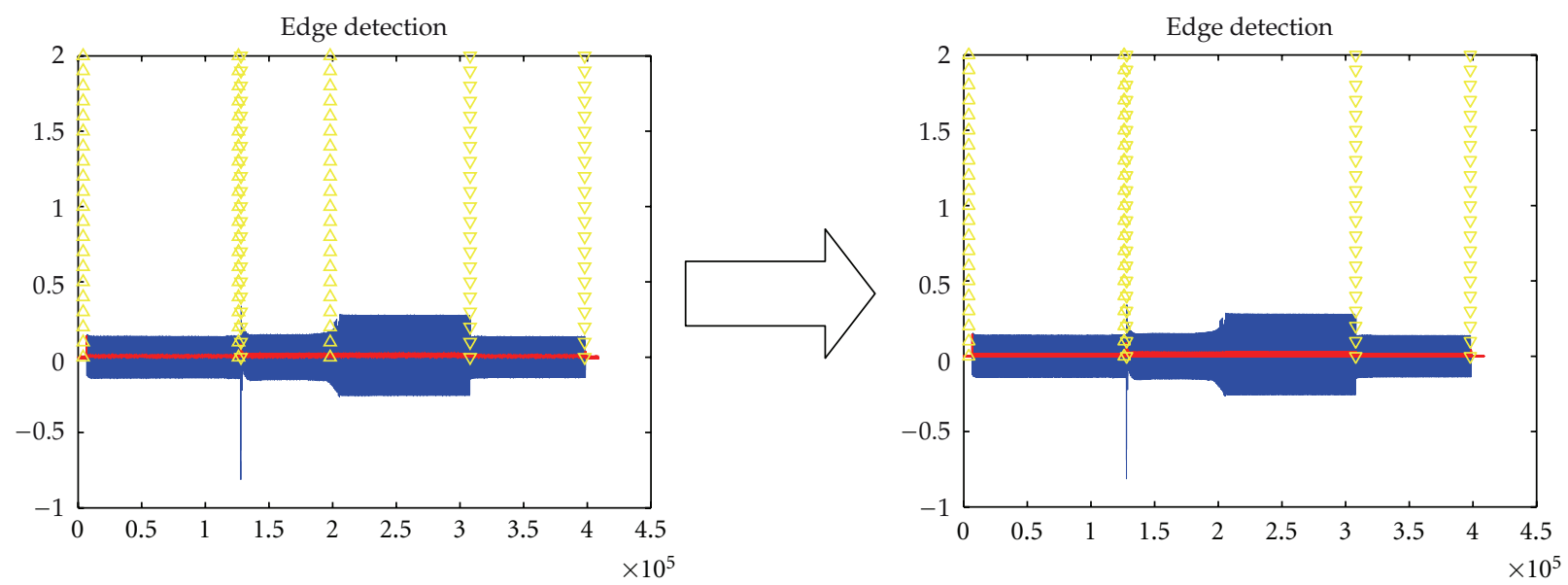

(a) Kettle + Microwave oven
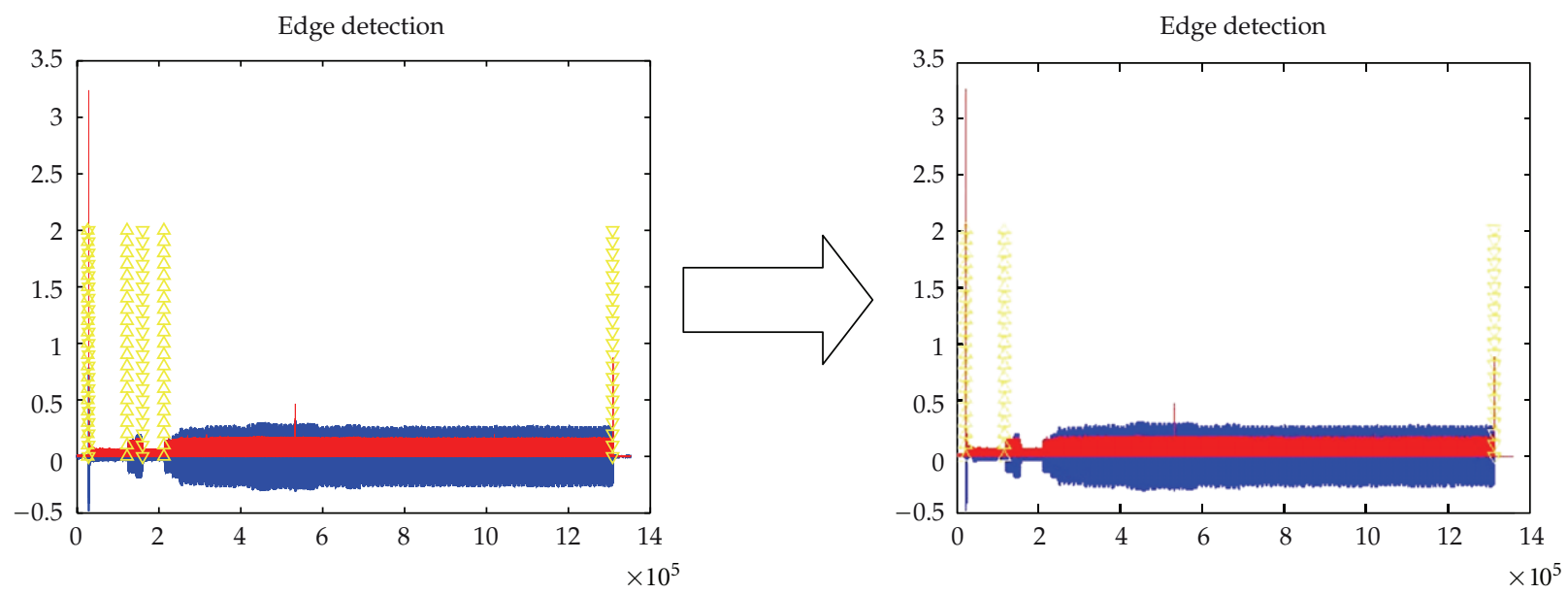

(b) Kettle + PC

FIgURE 5: Examples of power signal edge analysis.

of our algorithm. Moreover, despite the training processes always take several minutes, it avoid a mess of pre-work on processing signatures. The testing processes are much quicker than previous methods because of employing comparatively a small quantity of points from load frequency features. However, we would like to indicate that the number of appliance is limited and no transient event is involved. The performance may come down when more devices and events are used in experiments.

\section{Conclusion}

This paper has proposed one approach of nonintrusive appliance load monitoring for electrical consumption managing. This approach can automatically detect the switch-on and switch-off events of domestic appliances and classify different appliances using load features and advanced algorithms, therefore, monitoring the house power consumption of individual devices. The new transient detection algorithm, in combination with turn-on and turn-off transient waveforms
TABLE 3: Results of 11 loads classification.

\begin{tabular}{|c|c|c|}
\hline \multirow[b]{2}{*}{ Load appliance } & \multicolumn{2}{|c|}{ Accuracy of features (500-currence) } \\
\hline & $\begin{array}{l}\text { Unprocessed } \\
\text { current data }\end{array}$ & $\begin{array}{c}\text { Current } \\
\text { harmonic data }\end{array}$ \\
\hline Dremel & $100 \%$ & $100 \%$ \\
\hline Fan heater & $97 \%$ & $100 \%$ \\
\hline Heater gun & $97 \%$ & $100 \%$ \\
\hline Kettle & $90 \%$ & $97 \%$ \\
\hline Laptop & $100 \%$ & $100 \%$ \\
\hline LCD & $100 \%$ & $100 \%$ \\
\hline Incandescent light & $97 \%$ & $97 \%$ \\
\hline Fluorescent light & $100 \%$ & $100 \%$ \\
\hline Mini PC & $100 \%$ & $100 \%$ \\
\hline $\mathrm{PC}$ & $100 \%$ & $100 \%$ \\
\hline Pedestal fan & $100 \%$ & $100 \%$ \\
\hline
\end{tabular}

analysis, is developed to detect the mutative power events. The load classification technique, which employs support 

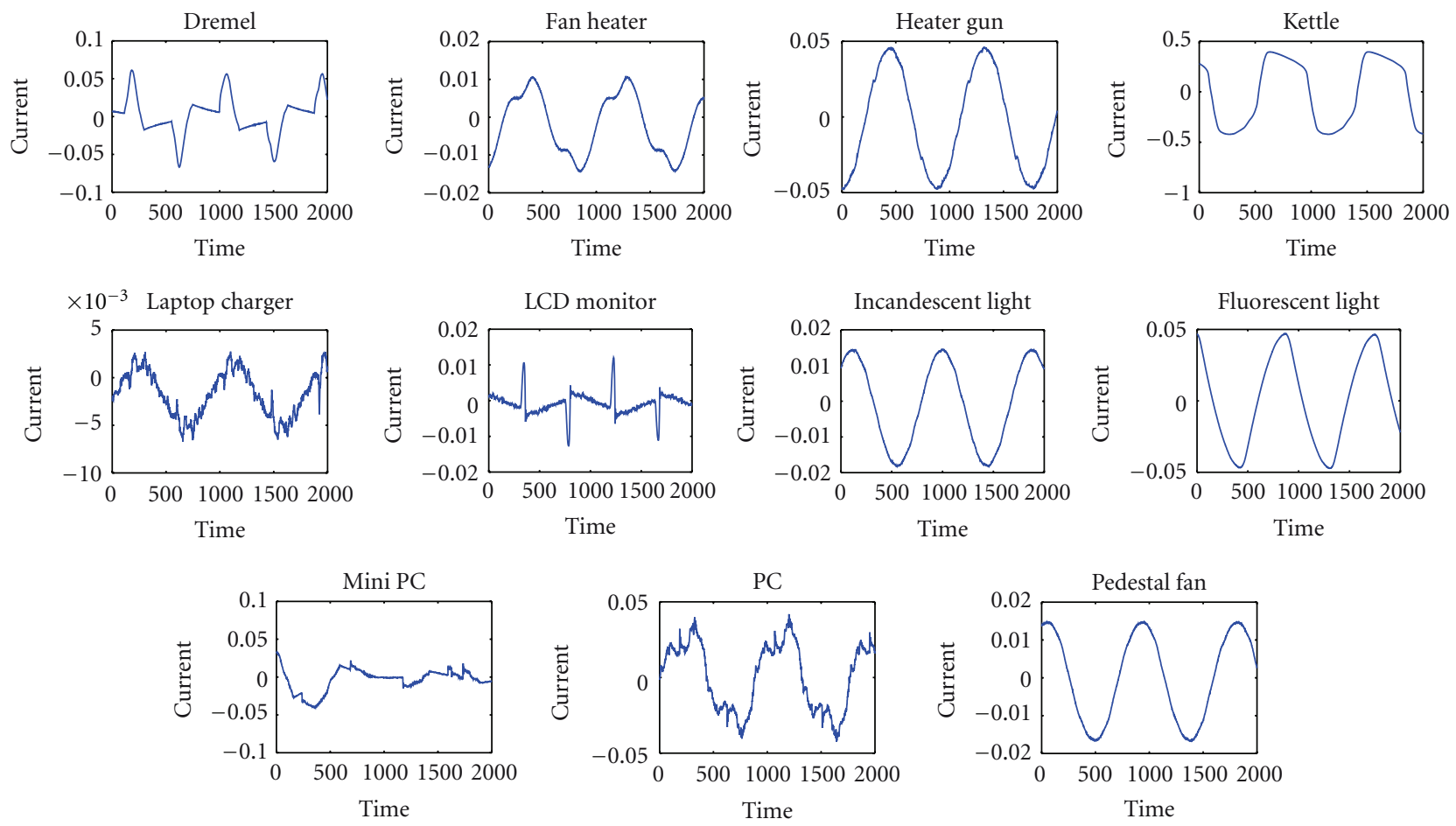

FIgURE 6: Examples of training set of unprocessed current data in time domain (steady state).
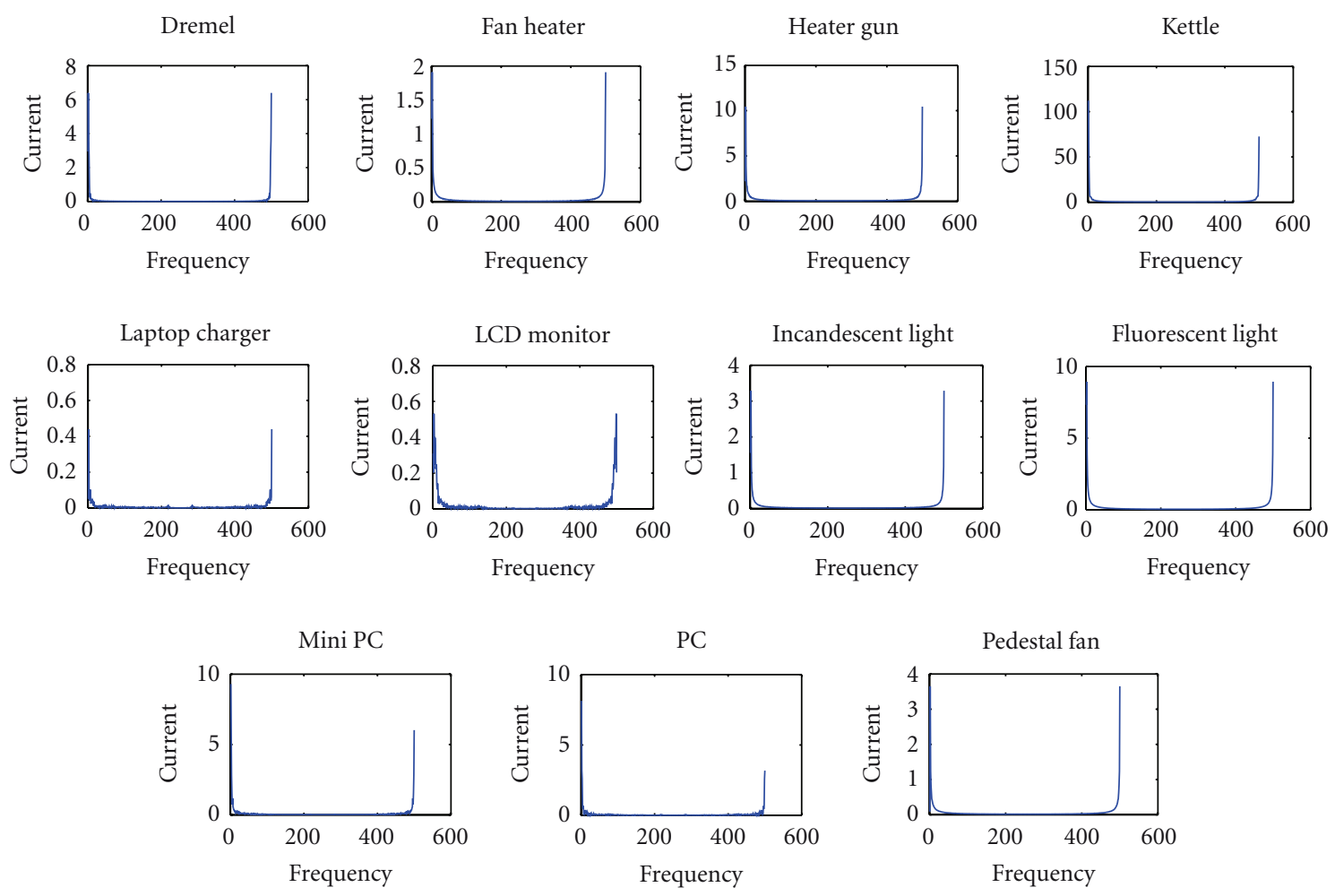

FIGURE 7: Examples of training set of current data in frequency domain (steady state). 
vector machine to recognize different appliances, is capable of identifying kinds of power loads with improved recognition accuracy and computational speed of NILM results. A recognition rate of higher than $90 \%$ has been achieved in recognizing various electrical devices. Our experiments have shown that recognition approaches based on support vector machines will be a trend for the process of residential load monitoring.

\section{References}

[1] G. Guo, S. Li, and K. Chan, "Face recognition by support vector machines," in Proceedings of the 4th IEEE International Conference on Automatic Face and Gesture Recognition, pp. 196-201, 2000.

[2] S. Du and T. Wu, "Support Vector Machines for Regression," Acta Simulata Systematica Sinica, TP18, CNKI:SUN: XTFZ.0.2003-11-022, 2003.

[3] S. Inagaki, T. Egami, T. Suzuki, H. Nakamura, and K. Ito, "Nonintrusive appliance load monitoring based on integer programming," Electrical Engineering in Japan, vol. 174, no. 2, pp. 1386-1392, 2011.

[4] S. Aoki, M. Onishi, A. Kojima, and K. Fukunaga, "Detection of a solitude senior's irregular states based on learning and recognizing of behavioral patterns," IEEJ, vol. 125, pp. 259265, 2005.

[5] C. Laughman, K. D. Lee, R. Cox et al., "Advanced nonintrusive monitoring of electric loads," IEEE Power and Energy Magazine, pp. 56-563, April 2003.

[6] J. Li, G. Poulton, and G. James, "Agent-based distributed energy management," in Proceedings of the 20th Australian Joint Conference on Advances in Artificial Intelligence, vol. 4830, pp. 569-578, December 2007.

[7] J. Li, G. Poulton, and G. James, "Coordination of distributed energy resource agents," Applied Artificial Intelligence, vol. 24, no. 5, pp. 351-380, 2010.

[8] Y. Guo, J. Li, and G. James, "Evolutionary optimisation of distributed energy resources," in Proceedings of the 18th Australian Joint Conference on Advances in Artificial Intelligence, vol. 3809, pp. 1086-1091, Sydney, Australia, December 2005.

[9] R. Li, J. Li, G. Poulton, and G. James, "Agent-based optimisation systems for electrical load management," in Proceedings of the 1st International Workshop on Optimisation in Multi-Agent Systems, pp. 60-69, Estoril, Portugual, May 2008.

[10] J. Li, G. James, and G. Poulton, "Set-points based optimal multi-agent coordination for controlling distributed energy loads," in Proceedings of the 3rd IEEE International Conference on Self-Adaptive and Self-Organizing Systems (SASO '09), pp. 265-271, San Francisco, Calif, USA, September 2009.

[11] J. Li, G. Poulton, G. James, and Y. Guo, "Multiple energy resource agent coordination based on electricity price," Journal of Distributed Energy Resources, vol. 5, pp. 103-120, 2009.

[12] G. W. Hart, "Nonintrusive appliance load monitoring," Proceedings of the IEEE, vol. 80, no. 12, pp. 1870-1891, 1992.

[13] S. B. Leeb, S. R. Shaw, and J. L. Kirtley, "Transient event detection in spectral envelope estimates for nonintrusive load monitoring," IEEE Transactions on Power Delivery, vol. 10, no. 3, pp. 1200-1210, 1995.

[14] M. C. Ferris and T. S. Munson, "Interior point methods for massive support vector machines," Tech. Rep. 00-05, Computer Sciences Department, University of Wisconsin, Madison, Wis, USA, 2000.
[15] C. J. C. Burges, "A tutorial on support vector machines for pattern recognition," Data Mining and Knowledge Discovery, vol. 2, no. 2, pp. 121-167, 1998.

[16] J. J. Moré and G. Toraldo, "Algorithms for bound constrained quadratic programming problems," Numerische Mathematik, vol. 55, no. 4, pp. 377-400, 1989.

[17] O. L. Mangasarian and D. R. Musicant, "Active set support vector machine classifiation," in Advances in Neural Information Processing Systems, T. Leen, T. Dietterich, and V. Tresp, Eds., vol. 13, pp. 577-583, MIT Press, Cambridge, Mass, USA, 2001.

[18] J. Li, S. West, and G. Platt, "Power decomposition based on SVM regression," in Proceedings of the 4th International Conference on Modelling, Identification and Control (ICMIC '12), pp. 1256-1261, Wuhan, China, June 2012.

[19] A. J. Bijker, X. Xia, and J. Zhang, "Active power residential non-intrusive appliance load monitoring system," in IEEE AFRICON Conference, pp. 1-6, September 2009.

[20] H.-H. Chang, K.-L. Chen, Y.-P. Tsai, and W.-J. Lee, "A new measurement method for power signatures of non-intrusive demand monitoring and load identification," in Proceedings of the 46th IEEE Industry Applications Society Annual Meeting (IAS '11), pp. 1-7, Orlando, Fla, USA, 2011.

[21] T. Onoda, H. Murata, G. Rätsch, and K.-R. Müller, "Experimental analysis of Support Vector Machines with different kernels based on non-intrusive monitoring data," in Proceedings of the International Joint Conference on Neural Networks (IJCNN '02), vol. 3, pp. 2186-2191, Honolulu, Hawaii, USA, 2002.

[22] R. Kadouche, B. Chikhaoui, and B. Abdulrazak, "User's behavior study for smart houses occupant prediction," Annals of Telecommunications, vol. 65, no. 9-10, pp. 539-543, 2010.

[23] S. R. Gunn, "Support vector machines for classification and regression," Faculty of Engineering, Science and Mathematics School of Electronics and Computer, University of Southampton, 1998.

[24] G. L. Grinblat, L. C. Uzal, H. A. Ceccatto, and P. M. Granitto, "Solving nonstationary classification problems with coupled upport vector machines," IEEE Transactions on Neural Networks, vol. 22, no. 1, pp. 37-51, 2011.

[25] J. Liang, S. K. K. Ng, G. Kendall, and J. W. M. Cheng, "Load signature studypart I: basic concept, structure, and methodology," IEEE Transactions on Power Delivery, vol. 25, no. 2, pp. 551-560, 2010.

[26] M. Zeifman and K. Roth, "Nonintrusive appliance load monitoring: review and outlook," IEEE Transactions on Consumer Electronics, vol. 57, no. 1, pp. 76-84, 2011.

[27] J. Canny, "A computational approach to edge detection," IEEE Transactions on Pattern Analysis and Machine Intelligence, vol. 8, no. 6, pp. 679-698, 1986.

[28] D. Demigny and T. Kamlé, "A discrete expression of canny's criteria for step edge detector performances evaluation," IEEE Transactions on Pattern Analysis and Machine Intelligence, vol. 19, no. 11, pp. 1199-1211, 1997.

[29] F. Truchetet, F. Nicolier, and O. Laligant, "Subpixel edge detection for dimensional control by artificial vision," Journal of Electronic Imaging, vol. 10, no. 1, pp. 234-239, 2001.

[30] I. Pitas and A. Venetsanopoulos, "Nonlinear mean filters in image processing," IEEE Transactions on Acoustics, Speech, and Signal Processing, vol. 34, no. 3, pp. 573-584, 1986.

[31] A. Benazza-Benyahia, J. C. Pesquet, and H. Krim, "A nonlinear diffusion-based three-band filter bank," IEEE Signal Processing Letters, vol. 10, no. 12, pp. 360-363, 2003.

[32] M. A. Schulze, "An edge-enhancing nonlinear filter for reducing multiplicative noise," in Nonlinear Image Processing VIII, 
E. R. Dougherty and J. Astola, Eds., vol. 3026 of Proceedings of SPIE, pp. 46-56, San Jose, Calif, USA, February 1997.

[33] H. Hwang and R. A. Haddad, "Multilevel nonlinear filters for edge detection and noise suppression," IEEE Transactions on Signal Processing, vol. 42, no. 2, pp. 249-258, 1994.

[34] O. Laligant and F. Truchetet, "A nonlinear derivative scheme applied to edge detection," IEEE Transactions on Pattern Analysis and Machine Intelligence, vol. 32, no. 2, pp. 242-257, 2010.

[35] L. Jiang, S. Luo, and J. Li, "An approach of household power appliance monitoring based on machine learning," in Proceedings of the 5th International Conference on Intelligent Computation Technology and Automation (ICICTA '12), pp. 577-580, January 2012.

[36] T. Joachims, "Making large-scale SVM learning practical," LS8-Report, University of Dortmund, 1998.

[37] C. Cortes and V. Vapnik, "Support-vector networks," Machine Learning, vol. 20, no. 3, pp. 273-297, 1995.

[38] A. B. Ji, J. H. Pang, and H. J. Qiu, "Support vector machine for classification based on fuzzy training data," Expert Systems with Applications, vol. 37, no. 4, pp. 3495-3498, 2010.

[39] S. Luo, Q. Hu, X. He, J. Li, J. S. Jin, and M. Park, "Automatic liver parenchyma segmentation from abdominal CT images using support vector machines," in Proceedings of the IEEE/CME International Conference on Complex Medical Engineering (ICME '09), p. 10071, Tempe, Ariz, USA, April 2009.

[40] L. Jiang, J. Li, S. Luo, and S. West, "Literature review of power disaggregation," in Proceedings of the IEEE International Conference on Modelling Identification and Control, pp. 38-42, 2011.

[41] R. Debnath and H. Takahashi, "Kernel selection for the support vector machine," IEICE Transactions on Information and Systems, vol. E87-D, no. 12, pp. 2903-2904, 2004. 

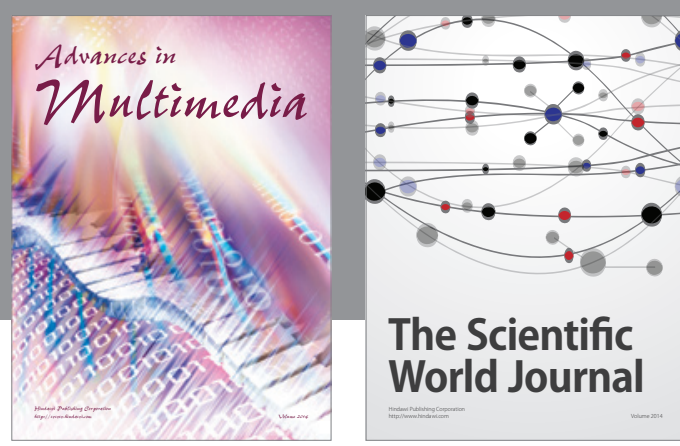

The Scientific World Journal
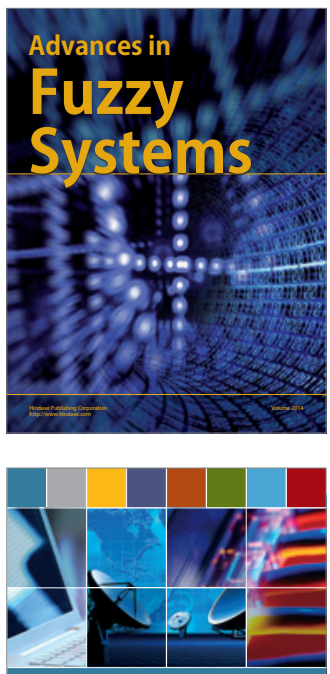

Computer Networks and Communications
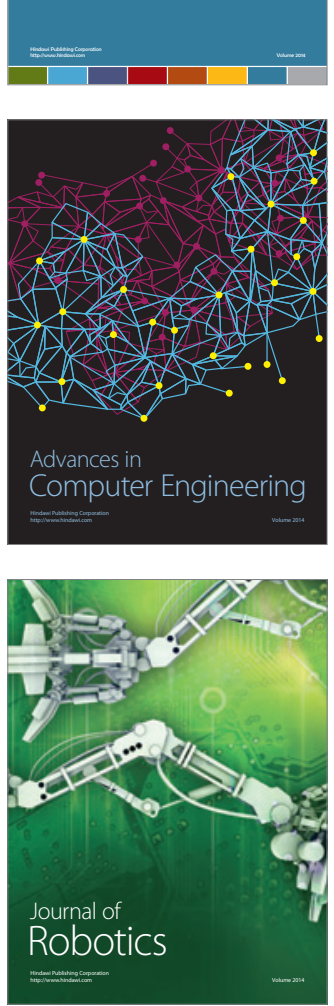
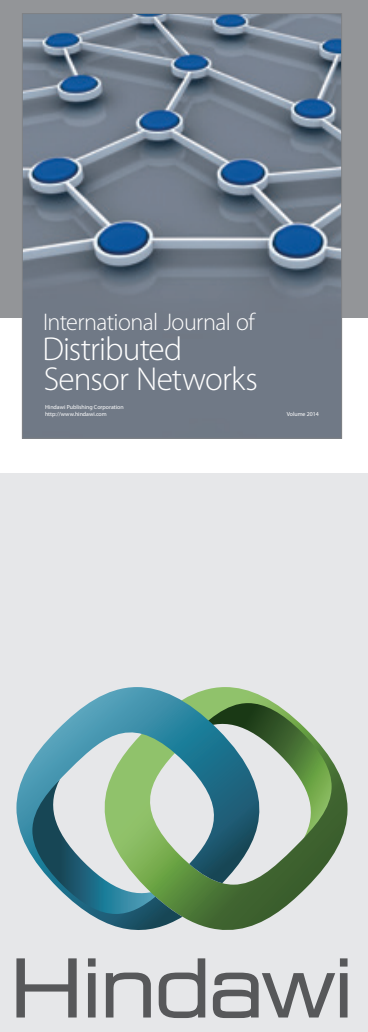

Submit your manuscripts at

http://www.hindawi.com
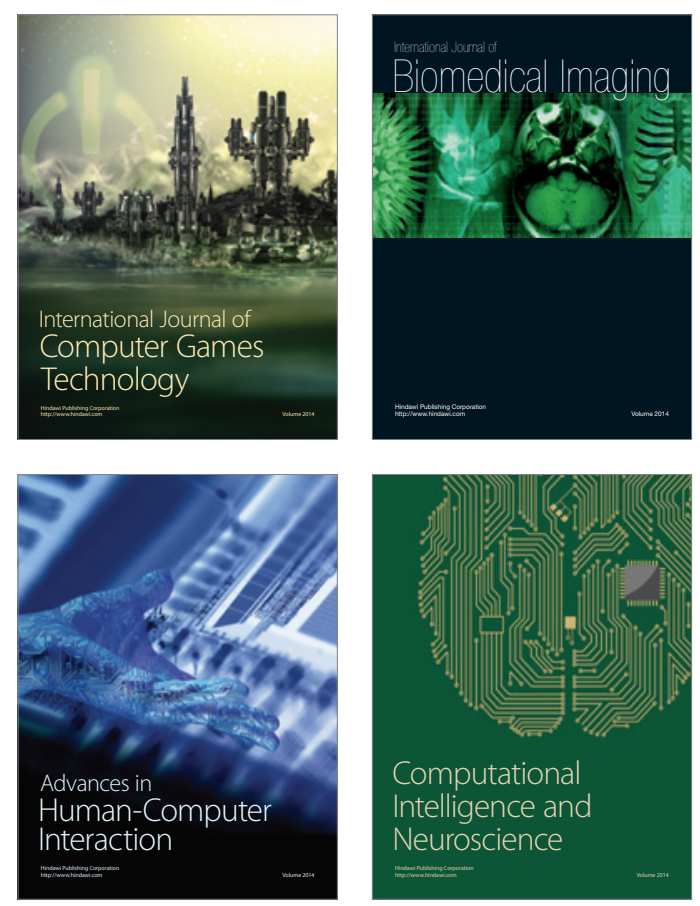
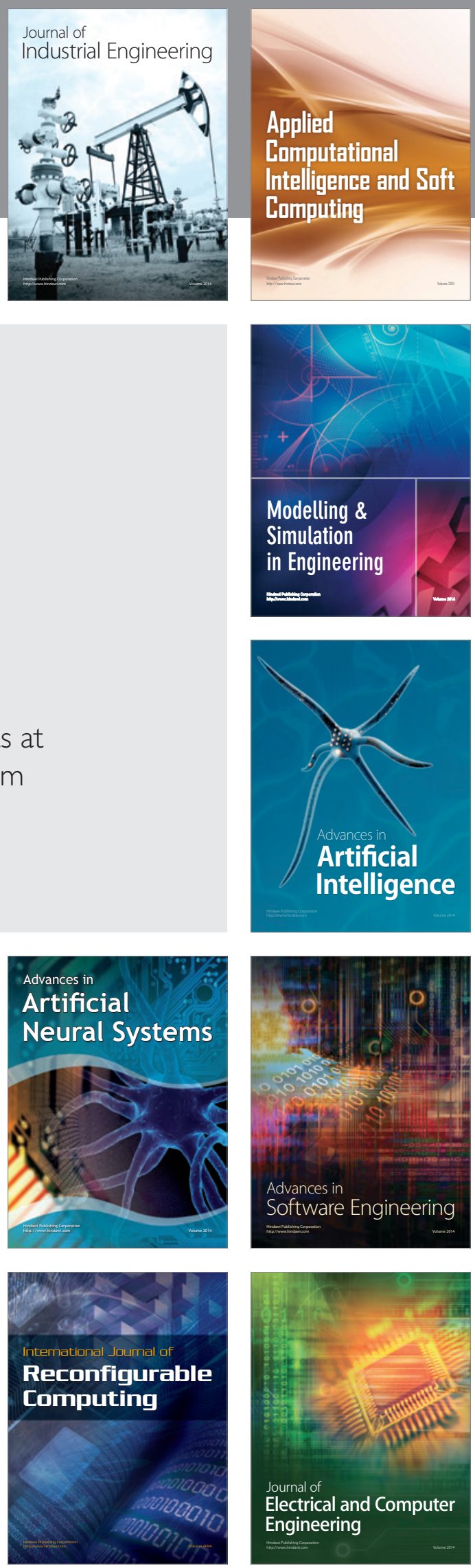